\title{
Re-Establishment of Health for Professionals in Higher Education During the COVID-19 Pandemic: Using Professors in an HIS as an Example
}

\author{
Pierre Lu \\ University of Texas Rio Grande Valley, USA
}

\begin{abstract}
The purpose of this study is to investigate faculty health and well-being (H\&W; SDG\#3) in a Hispanic Serving Institution (HSI) and how these professionals seek to re-establish their health during the COVID-19 pandemic. Recent research has shown that H\&W concerns from COVID-19 are much higher among Hispanics than Whites (Pew Research Center, 2020), and COVID-19 is affecting Hispanics at an alarming rate (CNN, 2020). Understanding how COVID-19 is affecting Hispanic faculty's $\mathrm{H} \& W$ is paramount as literature has shown that faculty H\&W are important to the success of their students, their profession, and their institutions of higher education. However, few studies have explored H\&W for faculty in an HSI. The study explores how these professionals re-establish their H\&W during the pandemic.
\end{abstract}

Keywords: Hispanic serving institution (HIS), covid-19, health and well-being

Article History

Received 13 September 2020

Accepted 31 October 2020

Published 31 January 2021

Available online 19 February 2021

https://doi.org/10.47544/johsk.2021.2.1.29

\author{
Corresponding Author \\ Pierre Lu \\ mingtsan.lu@utrgv.edu \\ Department of Health \& Human Performance \\ College of Health Profession \\ University of Texas Rio Grande Valley, Texas, USA
}

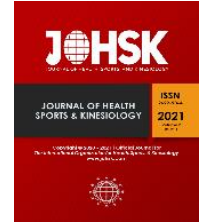

\section{Methods}

The study takes place in an HSI in South Texas. Survey method with convenience sampling ( $\mathrm{n}=50$ ), followed by one-on-one in-depth interviews with purposive sampling $(n=5)$ are conducted. Based on the Hetler's H\&W model and referenced to the Travis' and Ardell's models, all aspects of H\&W are inquired (physical, emotional, social, spiritual, vocational, and intellectual health). Quantitative data are analyzed using statistical methods.

\section{Results \& Conclusion}

$86 \%$ of participants reveal that this pandemic has decreased their H\&W. $75 \%$ of participants indicate they thought about building or re-establishing their $\mathrm{H} \& \mathrm{~W}$. However, only $30 \%$ of participants reveal they actually implement their H\&W plans. Qualitative data are analyzed using thematic analysis methods. Several themes emerge: (1) H\&W challenges faculty face, such as increased stress from work, family, and children at home; (2) their awareness and attempts of re-establishment of $\mathrm{H} \& \mathrm{~W}$, such as needs to be more organized, eat better, and exercise more; (3)

\section{Journal of Health, Sports, \& Kinesiology | ISSN 2692-9864 | www.johsk.com}


| 2021 | Volume 2 | Issue 1 | The Journal of Health, Sports, and Kinesiology |

recommendations from faculty that echo UN's H\&W tips, such as of staying home more, keeping social distancing, enjoying things in life, sleeping, exercising, having balanced diet, and a positive mental attitude.

\section{Journal of Health, Sports, \& Kinesiology | ISSN 2692-9864 | www.johsk.com}

